\title{
CRITICAL FLUCTUATIONS IN THE BREAKDOWN OF DISORDERED SYSTEMS
}

\author{
Alberto Petri \\ Istituto di Acustica "O.M. Corbino", Consiglio Nazionale delle Ricerche \\ via del Fosso del Cavaliere 100, 00135 Rome, Italy \\ and \\ Dipartimento di Fisica e Istituto Nazionale di Fisica della Materia \\ Università La Sapienza, P.le A. Moro 2, 00185 Rome, Italy
}

\begin{abstract}
In this paper some critical aspects of the behaviour of breaking lattices subject to slow driving forces are briefly reviewed. In particular, fluctuations in the response to the variation of external parameters are discussed.
\end{abstract}

\section{INTRODUCTION}

Antonio Coniglio's contribution to the undertstanding of rupture phenomena in disordered systems is twofold. On one hand he has contributed indirectly to the field, by furnishing fundamental knowledge indispensable for dealing with dilute networks, like e.g. the exponent for the scaling of the density of cutting bonds in a percolating lattice[1. On the other hand he has also pointed out important properties of the response of these systems, such as the multifractality of currents in diluted[2] and two component [3] resistor networks. These studies have continued in the observation, investigation and explanation of a large quantity of scaling properties in breaking lattices 4 , 5. and the great amount of work done in this field has shown that scale invariance is a fundamental property of rupture and breakdown phenomena of disordered systems 4 4 . Indeed, disorder plays a fundamental role in generating this kind of behaviour [6].

Scale invariance has been found to be also a more general characteristic of the dynamical response of these systems to the variation of some external parameter, and also a characteristic of the fluctuations of their response. For this reason, the adjective "critical" is often used, in analogy with the fluctuations of thermal systems at the critical point. In the following we shall mainly focus on this aspect of the breakdown phenomena and will consider in particular those situations in which an external perturbation (driving) is applied slowly with respect to the characteristic relaxation times of the system. After briefly reviewing some experimental results (Sec 2.), we shall describe how some of the observed features are reproduced by lattice models (Sec 3.), and some current points of view on the subject are (Sec 4.).

\section{SOME EXPERIMENTAL FACTS}


due to Mogi[7]. With the aim of verifying the validity of the Gutenberg Richter law also at scales much smaller than those involved in an earthquake, he designed and performed some original experiments where a controlled pressure was applied to disks made of a mixture of resin and hard grains. He observed that, by increasing the pressure, elastic waves were released from some localised region within the sample. By recording the maximum wave amplitude of each series Mogi was able to show that the relation between the observed amplitudes and their frequency of occurrence was of the algebraic type, the one known as the Ishimoto and Iida's law in the seismological field. These findings, besides to show in a quantitative way that earthquakes and fractures have common features, demonstrated the intrinsic critical nature and the importance of disorder in the response of a medium to the external perturbations.

Laboratory experiments can well reproduce not only the critical features observed in distribustion of the energy released by earthquakes, but also those observed in the distribution of times. In 1968 Scholz succeeded in reproducing Omori's power law describing the number of aftershocks observed in a time $t$ after a main event $[$ ]. By analysing the acoustic emission from a fracturing basalt rock he also coonfirmed the validity of the Gutenberg-Richter law [9]. In subsequent investigations Hirata 10] showed that Omori's law holds also for generic microfracturing processes, at least for times large enough after the main event.

Thanks to the advances in technology and informatics, experiments in this fields continue to bring new results. By collecting and analyzing acoustic emission signals from concrete-like samples, we have shown 11] that power laws not only describes the frequency distribution of maximum amplitudes in the aftershock series, but also the amplitude distibution of the entire time series, as well as the time lags distribution between consecutive meaningful events of acoustic emission. We have found in particular that the energy (proportional to the squared amplitude) is distributed according to $N(E) \simeq E^{-\delta}$ with $\delta$ around 1.3, while for time lags one has $P(t) \simeq t^{-\zeta}$ with $\zeta$ approx 1.6 [12]. In addition, lacking of characteristic scales in the fluctuations of response has been brought into evidence by measuring the power spectrum and the autocorrelation of the time series. The power spectrum has been found of the $1 / f^{\gamma}$ form, with $\gamma \simeq 0.6[11$, 12.

In more recent experiments Ciliberto and coworkers 13, 14 found $\delta \simeq 1.25$ for the energy scaling and conjectured its universality, whereas $\zeta$ was found to depend on the applied external stress. Acoustic emission recorded during pressurization of spherical tanks yielded still more evidences of the critical nature of breakdown phenomena and revealed the presence of logarithmic oscillations in the power laws 15. Finally, Maes and coworkers 16] observed scale invariance in acoustic emission amplitudes, time lags, and spatial distance between consecutive events also in a cellular glass, where they found $\zeta=1.3$, but $\delta=2$.

Another important evidence of the critical response of disordered media concerns the roughness of the fracture surfaces 17]. After Mandelbrot and coworkers measured self-affinity properties in the fracture surface of some steels, similarity in the exponents $(\approx 0.8)$ characterizing many different materials has been pointed out 18, 19. Further experiments have shown later that also 0.5 is observed[20]. According to some evidences lower values seem to characterize slow producing cracks and roughness at small scales, 


\section{LATTICE MODELS}

Existence of critical fluctuations in the response of model systems was firstly observed [22] [23] in the fibre bundle model [24], where an external applied stress is evenly shared by a stretched bundle of fibers. The elastic modulus is the same for all the fibers, but each one can stand a different, finite, amount of stress. The system is initially unloaded, then stress is applied in order to break the weaker fiber of the bundle. The excess stress is shared by the other fibres, that therefore become more prone to break; whenver some fibre exceeds its own failure threshold it also breaks and stress is redistributed again. When no more fibres break stress is increased again, up to break the weakest of the survived fibers, and so on until all the fibres of the bundle are broken. Each breaking process is carried on at constant stress. By exact calculation Hansen and Hammer were able to show that as the applied stress is increased from zero to the global failure value, there is a probability $P(s) \propto s^{-\alpha}$ that $s$ fibers break in correspondance of the same value of applied stress. The exponent was found to be $\alpha=5 / 2$ and largely independent of the statistical distribution of fiber strenghts. Hansen and Hammer also observed power law distributions for "avalanches' of broken fuses in the numerical simulation of a square resistor network where, in analogy with he fiber bundle model, each fuse posseses the same conductance, but can stand different maximum current. When a fuse finds itself above its own threshold of rupture, it breaks and the excess current is shared by the other fuses. It may happen therefore that some other fuse burn in turn, and so on. They found numerically $\alpha=2.7$ for this system, very close to $5 / 2$.

Presence of similar behaviour was later observed in system with vector elasticity by numerical simulations of the Born model on a triangular lattice 25. In this model sites interact via the potential

$$
V_{i j}=(\alpha-\beta)\left[\left(\vec{u}_{i}-\vec{u}_{j}\right) \cdot \vec{r}_{i j}\right]^{2}+\beta\left[\vec{u}_{i}-\vec{u}_{j}\right]^{2},
$$

where $\vec{u}_{i}$ is the displacement vector of site $i$ from equilibrium, $\vec{r}_{i j}$ is the unit vector between the initial equilibrium position of sites $i$ and $j$, and $\alpha$ and $\beta$ are force constants. In the case of Ref. [25] avalanches were also triggered by a corrosion mechanisms, according to which the bonds neighbouring a broken bond are weakened. Such a mechanism enhances the critical properties of the system [26], and the related exponent was found to be $\alpha=2.0$, the same found in the experiments on the cellular glass 16.

The power law distributions mentioned above are computed by considering all the avalanches occurring during the life of the system. One can also consider what happens for a given value of the external solicitation (stress, strain, current) $\sigma$ when averaging over many realizations. For the fibre bundle it has been shown that the following scaling form holds 23]:

$$
P(s, \sigma)=f\left(\frac{s}{s_{0}}\right) s^{-\tau}
$$

where

$$
s_{0} \approx\left(\sigma_{c}-\sigma\right)^{-\kappa}
$$

and $\tau=1.5$ and $\kappa=1 ; \sigma_{c}$ represents the critical value of stress at which the network definitively tears. Thus, if one cumulates all the avalanches from the beginning, $\sigma=0$, to the end of the system life, $\sigma=\sigma_{c}$, one observes 
with $\alpha=\tau+1 / \kappa=2.5$. Zapperi at al. 31 succeeded to show that within the effective medium approximation the fuse network obeys the same scaling of the fibre bundle model, and that the ensemble averaged burst size $\langle s\rangle$ diverges at criticality as

$$
\langle s\rangle \approx\left(\sigma_{c}-\sigma\right)^{-\gamma}
$$

with $\gamma=1 / 2$. These results have been confirmed numerically both on square fuse networks 31 and on elastic networks with central and bond bending potentials 32.

A way for quantifying the vicinity of a system to some critical point is to define a branching ratio $\rho$ for the process as the probability for the breaking of a bond to give rise to the breaking of another bond. In analogy with other processes [33] at the critical point $\rho=1$. We have evaluated $\rho$ for the Born potential on a triangular lattice [26] by averaging over many realizations the number of broken bonds at fixed values of the external driving (stress or strain) $\langle s\rangle$. This quantity is related to $\rho$ by:

$$
\rho=\frac{\langle s\rangle-1}{\langle s\rangle}
$$

and therefore

$$
\rho \approx 1-\left(\sigma_{c}-\sigma\right)^{-\gamma}
$$

Numerical simulations show that different types of driving produce different behaviours,

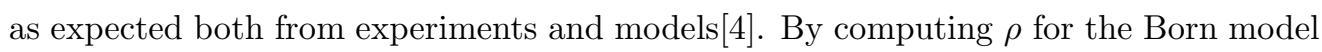
we have shown in particular that breaking the bonds by driving with strain makes the system less critical than breaking them by driving with stress, and that the presence of corrosion mechanisms leads closer to criticality.

It must be stressed that the scale invariance for the breaking of bonds in model systems cannot be directly assimilated to that of acoustic emission. In fact, the latter carries an energy content that is not included in the broken bonds counting. Selfsimilarity of the energy bursts in lattice models only holds at comparable values of the external driving applied 25], or when special conditions are imposed to the system 34].

Self affine properties of the fracture surface are rather well reproduced by model simulations. In particular, numerical results suggest that small self-affinity exponents (around 0.5) are related to slowly moving cracks, whilst exponents close to 0.8 are associated with fast cracks [35, 36]. Some different exponents, like 0.42 have been also found for slowly developping cracks in two dimensions [37], but also if simulations well reproduce generally the experimental observations, there is no a comprehensive theory for all these behaviours (for a recent review on dynamic fractures see Ref. 38].

\section{POINTS OF VIEW}

A coherent description of breakdown phenomena and of their critical properties is lacking. One possibility is to relate them to the Self Organized Criticality (SOC) 27. In this picture the SOC state corresponds to the final breakdown. Another tempting possibility is that of exploiting the analogies with critical phenomena for setting up a thermodynamic-like description of the fracturing process. Within this framework Zapperi et al. 31] suggested to choose the elastic moduli (or the conductance for the fuse 
in usual first order transitions, would originate in this case from the presence of long range interactions; In different situations these have been shown to give rise to fractal fluctuations in the nucleation process 28. The mean field picture, from which this interpretation is derived, does not describe all the features of the breaking process in euclidean lattices: pheraps the most relevant shortcoming is that the theory does not predict the scaling of the breaking stress distribution with the system size 29. Moreover, Sornette [39, 40] and da Silveira [41, 42 have found that the choice of different disorder distributions can select between continuous and discontinuous transition, with a tricritical point separating the two behaviours in the phase diagram of the system. Finally, continuous breakdown transition can also be observed when constant strain instead of constant stress driving is applied to the lattice 43].

In conclusion we may say that in spite of the observation of critical fluctuations in many breakdown phenomena, the physical mechanisms at their origin are still far from clear and that it seems necessary to take into account both irreversibility and non self-averaging in order to come to a satisfactory explanation of these complicated non-equilibrium processes.

\section{REFERENCES}

\section{References}

[1] A. Coniglio, J. Phys. A10 (1977) L211.

[2] L. de Arcangelis, S. Redner and A. Coniglio, Phys. Rev. B31 (1985) 4725.

[3] L. de Arcangelis and A. Coniglio, J. Stat. Phys. 48 (1987) 935.

[4] H. J. Herrmann and S. Roux, Statistical Models for the Fracture of disordered Media (North-Holland, Amsterdam, 1990), and refs. therein.

[5] B. K. Chakrabarti and L. G. Benguigui, Statistical Physics of Fracture and Breakdown in disordered Systems (Clarendon Press, Oxford, 1997), and refs. therein.

[6] B. Kahng, G.G. Batrouni, S. Redner, L. de Arcangelis, H. J. Herrmann, Phys. Rev. B37 (1988) 7625.

[7] K. Mogi, Tectonophysics 5 (1967) 35, and references therein.

[8] C.H. Scholz, Bull. Seismol. Soc. Am. (1968) 581117.

[9] C.H. Scholz, Bull. Seismol. Soc. Am. (1968) 58399.

[10] T. Hirata, J. Geophys. Res. 92 (1987) 6215.

[11] A. Petri, G. Paparo, A. Vespignani, A. Alippi, and M. Costantini, Phys. Rev. Lett. 73 (1994) 3423.

[12] A. Vespignani, A. Alippi, M. Costantini, G. Paparo, A. Petri, Fractals 3 (1995) 839.

[13] A. Guarino, L. Bellon, S. Ciliberto, Phys. Rev. Lett. 79 (1997) 3202.

[14] A. Guarino, A. Garcimartin and S. Ciliberto, Eur. Phys. J. B6 (1998) 13.

[15] D. Sornette and V.J. Andersen, Eur. Phys. J. B1 (1998) 353. 
[16] C. Maes, A. Van Moffaert, H. Frederix and H. Staruven, Phys. Rev. B57 (1998) 4987.

[17] B.B Mandelbrot, D.E Passoja, and A.J. Paullay, Nature 308 (1984) 721.

[18] E. Bouchaud, G. Lapasset and J. Planès, Europhys. Lett. 13 (1990) 73.

[19] K.J. Maloy, A. Hansen, E.L. Hinrichsen and S.Roux, Phys. Rev. Lett. 68 (1992) 213.

[20] V.Y Milman, R. Blumenfeld, N.A Stelmashenko and R.C Ball, Phys. Rev. Lett. 71 (1993) 204.

[21] P. McAnulty, L.V. Meisel and P.J Cote, Phys. Rev. A46 (1992) 3523.

[22] D. Sornette, J. Phys A: Math. Gen. 22 (1989) L243.

[23] C. Hemmer and A. Hansen, J. Appl. Mech. 59 (1992) 909.

[24] H.E.Daniels, Proc. R. Soc. London A183 (1945) 405.

[25] G. Caldarelli, F. di Tolla and A. Petri, Phys. Rev. Lett. 77 (1996) 2503.

[26] G. Caldarelli, C. Castellano and A. Petri, Physica (1999) A270 15.

[27] P. Bak, C. Tang and K. Wiesenfeld, Phys. Rev. Lett. 59 (a987) 381.

[28] T.S. Ray and W. Klein J. Stat. Phys. 61 (1990) 891.

[29] G. Caldarelli and A. Petri, Phys. Rev. Lett. 83, (1999) 1483.

[30] E. Bouchaud, J. Phys C: Cond. Matt. 9 (1997) 4319.

[31] S. Zapperi, P. Ray, H. E. Stanley and A. Vespignani, Phys. Rev. Lett. 78 (1997) 1408.

[32] M. Acharaya, P. Ray and B.K. Chakrabarty, Phys. Rev. E53 (1996) 140.

[33] O. Kinouchi, S.T.R. Pinho and C.P.C. Prado, Phys. Rev. E58 (1998) 3997.

[34] S. Zapperi, A. Vespignani and H.E. Stanley, Nature 388 (1997) 658.

[35] B. Chakrabarti and B.K. Chakrabarti, Physica A270 (1999) 21.

[36] A. Parisi, G. Caldarelli and L. Pietronero, Europhys. Lett. 52 (2000) 304.

[37] A. Nakano, R.K Kalia and P.Vashista Phys. Rev. Lett. 75 (1995) 3138.

[38] J. Fineberg and M. Marder, Phys. Rep. 313 (1999) 1.

[39] J.V Andersen, D. Sornette and K. Lung, Phys. Rev. Lett. 78 (1997) 2140.

[40] D. Sornette, K.-T. Leung and J. V. Andersen, Phys. Rev. Lett. 80 (1998) 3158.

[41] R. da Silveira, Phys. Rev. Lett. 80 (1998) 3157.

[42] R. da Silveira, Am. J. Phys. 67 (1999) 1177.

[43] G. Caldarelli, C. Castellano and A. Petri, Phil. Mag. B79 (1999) 1939. 